\title{
Updated Checklist and Global Diversity of Chaeteessidae, Mantoididae, Metallyticidae, Acanthopidae, Amorphoscelididae and Sibyllidae (Mantodea: Insecta)
}

\author{
Shveta Patel, Rajendra Singh \\ Department of Zoology, D.D.U. Gorakhpur University, Gorakhpur, U.P., India
}

\begin{abstract}
The praying mantids (Order Mantodea, Class Insecta) are a group of over 2500 carnivorous polyneopteran insects distributed in tropics and subtropics of the world, from the rainforest to the desert ground. The order Mantodea comprises over 20 families, out of which the global distribution of six families: Chaeteessidae, Mantoididae, Metallyticidae, Acanthopidae, Amorphoscelididae and Sibyllidae were provided in this compilation. Chaeteessidae includes just one extant genus with 6 species and Mantoididae comprises two genera with 12 species and both are distributed in Neotrpical South America. Metallyticidae includes just one genus containing 5 species inhabiting in Southeast Asia. Acanthopidae, commonly known as dead-leaf mantids or boxer mantids, consists of 14 genera and 96 species and are exclusively distributed in Neotropics of South America. It includes 3 subfamilies, Acanthopinae (8 genera, 53 species), Acontistinae (5 genera, 40 speies), and Stenophyllinae (1 genus, 3 species). Amorphoscelidae, commonly known as bark mantids, are includes three subfamilies, Amorphoscelinae (5 genera, 62 species), Paraoxypilinae (8 genera, 30 species), and Perlamantinae (2 genera, 3 species) with 15 genera and 95 species/subspecies distributed in the Tropical and Southern regions of Africa over to the Middle East and the Oriental region, including New Guinea. Sibyllidae is exclusively African family including only three genera and 17 species.
\end{abstract}

Keywords: Chaeteessidae, Mantoididae, Metallyticidae, Acanthopidae, Amorphoscelididae, Sibyllidae, world distribution, praying mantis, checklist.

\section{INTRODUCTION}

The praying mantids belong to the order Mantodea which is often considered as minor order of class Insecta as it constitutes a group of over only 2500 species [1]. Mostly, mantids are skilled opportunitstic ambush predators, remain stationary until suitable prey passes at which point they use their raptorial forelegs to catch their prey. However, some species are actively stalk cryptic conspecifics [2]. Although they usually prey on other insects, they are known to occasionally take small vertebrates such as lizards, frogs and hummingbirds [3]. Because of their efficient hunting techniques, they play a vital role in the natural control of insect pests [4]. They are predatory mostly inhabiting in tropical and subtropical habitats of the world, from the highly humid rainforest to the desert ground [5-10]. Its phylogeny was discussed by many workers in past [11-15].

Over the last 100 years there have been several publications on the Mantodea of the world [16-29], however, the most recent papers were published after eighties of twentieth century and since then there have been numerous changes to the nomenclature even at suprageneric levels because of complexity and plasticity of their external morphology that creat major obstacles for recognising natural groups [11, 12, 30-36].

Presently, the order Mantodea comprises over 20 families, out of which the family Mantidae alone includes 1255 species/subspecies described under 188 genera included in 21 subfamilies [37]. This paper the world mantids of six families, viz. Chaeteessidae, Mantoidae, Metallyticidae, Acanthopidae, Amorphoscelidae, and Sibyllidae are catalogued.

Chaeteessidae and Mantoididae include small, stout-built, very active species from tropical areas of South, Central and southern North America and unlike most mantids, they are active hunters that chase their prey [38]. This behaviour, along with their plesiomorphic morphology, they are considered as two of the basal-most extant taxa in Mantodea [12, 39-42]. Chaeteessidae includes just one extant genus: Chaeteessa Burmeister, 1838 with six species and Mantoididae comprises two genera: 
Mantoida Newman, 1838 with 11 species and Paramantoida Agudelo, 2014 with one species. These genera have never been reviewed taxonomically and the current taxonomic literature allows the identification of only a handful of species. They are distributed only in South and Central America [43-46].

Like above two families, Metallyticidae includes just one genus, Metallyticus Westwood, 1835 which is one of the most fascinating praying mantids but little is known of its biology [38, 47]. It comprises only five species. Metallyticus is restricted to the Oriental region [25, 29, 33]. Most of the specimens collected in the more than 170 years since the description of the genus were found throughout the Malayan and Indonesian regions.

Acanthopidae, commonly known as dead-leaf mantids or boxer mantids is relatively large group, consists of 14 genera and 96 species and are exclusively distributed in neotropical ecozone of America. They are mostly dead-leaf mimics and the females hang upside down from twigs and branches resembling dry, curled leafs [48-50]. In Acanthopidae, three subfamilies are included: Acanthopinae (8 genera, 53 species), Acontistinae (5 genera, 40 species), and Stenophyllinae (1 genus, 3 species). However, considering the morphological diversity among these three lineages and the lack of definite autopomorphies [13], including these taxa as members of a single family is suspect [15]. Acanthopinae includes some of the most cryptic and bizarrelooking mantids of the neotropics [51]. The Acanthopidae are widely distributed from central Mexico south to the Atlantic rainforest of southern Brazil [52-58].

The member of the family Amorphoscelidae, commonly known as bark mantids, are small to medium in size, mostly with cryptic colour of tree-bark and some mimic ants. They hunt on ground or on tree trunks. Usually females are apterous or brachypterous while males are fully winged. In most of them, sexual dimorphism is distinct. Amorphoscelidae includes three subfamilies: Amorphoscelinae (5 genera, 62 species), Paraoxypilinae (8 genera, 30 species), and Perlamantinae (2 genera, 3 species) with 15 genera and 95 species/subspecies. Most of the Amorphoscelinae are distributed in the Tropical and Southern regions of Africa over to the Middle East and the oriental region, including New Guinea. All members of Amorphoscelinae are very unique within the order Mantodea as they are characterided by the absence of any spination of the fore femora and fore tibiae [59-61], whereas, Paraoxypilinae are distributed in Australia and nearby. Stenophyllinae are small group of mantids distributed in Western Africa.

Sibyllidae is exclusively African family including only three genera and 17 species. They can be easily identified by having a long and thin prothorax with lateral and dorsal projections. The head bears an erect process with four sideways spikes. The taxonomy of Sibyllidae was thoroughly reviewed by Roy [62].

Most of the distributional records are scattered in literature [53]. The checklists of Mantodea of different countries/continents/ecozones are published in recent past by several authors $[6,10,29,33$, 53, 63-76]. In the present compilation, only valid name of the species is presented after critical scrutiny of literature. Hence, the present article will help in solving an up-todate listing of the mantid species of 6 families of Mantodea, viz. Chaeteessidae, Mantoididae, Metallyticidae, Acanthopidae, Amorphoscelididae and Sibyllidae. In preparing of this checklist, recent literatures (published up to October, 2016) were scrutinized for synonymy along with the information available at two websites (http://mantodea.speciesfile.org and http://www.gbif.org/species) accessed on 25 October, 2016.

\section{Global Check-List}

Following is the checklist of the six families of Mantodea. Synonymy of the taxa were avoided and for that literature published in recent past may be consulted [6, 15, 33, 51-54, 61, 63, 66, 78-80].

\section{Family: Chaeteessidae \\ 1. Subfamily: Chaeteessinae \\ 1. Genus: Chaeteessa Burmeister, 1838}

1. Chaeteessa burmeisteri Giebel, 1862 [Brazil]

2. Chaeteessa caudata Saussure, 1871 [Brazil, Costa Rica, Venezuela]

3. Chaeteessa filata Burmeister, 1838 [Brazil, Surinam]

4. Chaeteessa nana Jantsch, 1995 [Brazil]

5. Chaeteessa nigromarginata Salazar, 2004 [Colombia]

6. Chaeteessa valida (Perty, 1833) [Brazil, Colombia, French Guiana] 
Updated Checklist and Global Diversity of Chaeteessidae, Mantoididae, Metallyticidae, Acanthopidae, Amorphoscelididae and Sibyllidae (Mantodea: Insecta)

\section{Family: Mantoididae \\ 1. Subfamily: Mantoidinae \\ 2. Genus: Mantoida Newman, 1838}

7. Mantoida argentinae La Greca, 1990 [Argentina]

8. Mantoida beieri Kaltenbach, 1957 [Argentina]

9. Mantoida brunneriana (Saussure, 1871) [Bolivia, Brazil, Colombia, French Guiana, Panama, Paraguay, Venezuela]

10. Mantoida burmeisteri (Giebel, 1862) [Brazil]

11. Mantoida fulgidipennis Westwood, 1889 [Brazil, Colombia, French Guina, Surinam, Trinidad \& Tobago, Venezuela]

12. Mantoida maya Saussure \& Zehntner, 1894 [Brazil, Florida Keys-USA, Mexico, Panama, Venezuela]

13. Mantoida nitida Newman, 1838 [Argentina, Bolivia, Brazil, Mexico, Venezuela]

14. Mantoida ronderosi La Greca, 1990 [Argentina, Brazil]

15. Mantoida schraderi Rehn, 1951 [Costa Rica, Panama, Trinidad]

16. Mantoida tenuis (Perty, 1833) [Argentina, Brazil, Colombia, Paraguay, Uruguay]

17. Mantoida toulgoeti Roy, 2010 [Neotropic-South America]

\section{Genus: Paramantoida Agudelo, 2014}

18. Paramantoida amazonica Agudelo, 2014 [North Amazon-Brazil]

\section{Family: Metallyticidae \\ 1. Subfamily: Metallyticinae \\ 4. Genus: Metallyticus Westwood, 1835}

19. Metallyticus fallax Giglio-Tos, 1916 [Borneo, Sumatra]

20. Metallyticus pallipes Giglio-Tos, 1917 [Borneo]

21. Metallyticus semiaeneus Westwood, 1889 [Borneo]

22. Metallyticus splendidus Westwood, 1835 [Borneo, Java, India, Malayasia, Moluccan Islands, Sarawak, Sumatra]

23. Metallyticus violaceus Burmeister, 1838 [Borneo, Java, India, Indonesia, Malasia, Myanmar, Philippines, Sumatra]

\section{Family: Acanthopidae \\ 1. Subfamily: Acanthopinae \\ 1. Tribe: Acanthopini}

5. Genus: Acanthops Serville, 1831

24. Acanthops bidens Hebard, 1922 [Colombia, Mexico]

25. Acanthops boliviana Chopard, 1916 [Bolivia]

26. Acanthops brunneri Saussure, 1871 [Brazil, Colombia]

27. Acanthops centralis Lombardo \& Ippolito, 2004 [Colombia, Costa Rica, Panama]

28. Acanthops chocoensis Salazar, 2005 [Colombia]

29. Acanthops contorta Gerstaecker, 1889 [Brazil, Peru]

30. Acanthops elegans Lombardo \& Ippolito, 2004 [Costa Rica, Guatemala]

31. Acanthops erosa Serville, 1839 [Brazil, French Guiana]

32. Acanthops erosula Stal, 1877 [Bolivia, Brazil, Ecuador, Panama, Peru]

33. Acanthops falcata Stal, 1877 [Brazil, Colombia, Ecuador, Guiana, Mexico, Panama, Venezuela, Trinidad]

34. Acanthops falcataria (Goeze, 1778) [Brazil, East Africa]

35. Acanthops fuscifolia (Olivier, 1792) [Colombia, French Guiana, Guiana]

36. Acanthops godmani Saussure \& Zehntner, 1894 [Colombia, Belize, Guatemala, Costa Rica, Mexico]

37. Acanthops onorei Lombardo \& Ippolito, 2004 [Ecuador]

38. Acanthops parafalcata Lombardo \& Ippolito, 2004 [Caribbean Islands, Trinidad \& Tobago]

39. Acanthops parva Beier, 1941 [Brazil]

40. Acanthops royi Lombardo \& Ippolito, 2004 [Ecuador]

41. Acanthops soukana Roy, 2002 [French Guiana]

42. Acanthops tuberculata Saussure, 1870 [Brazil, Colombia, French Guiana, Guina, Peru] 


\section{Genus: Astollia Kirby, 1904}

43. Astollia chloris (Olivier, 1792) [Surinam]

\section{Genus: Beieracanthops Rafael, 2014}

44. Beieracanthops amazonica (Beier, 1930) [Brazil, French Guiana]

45. Beieracanthops rafaeli Rafael, 2014 [Brazil]

\section{Genus: Decimiana Uvarov, 1940}

46. Decimiana bolivari (Chopard, 1916) [Brazil, Paraguay]

47. Decimiana clavata Ippolito and Lombardo, 2004 [Brazil]

48. Decimiana hebardi Lombardo, 2000 [Argentina, Bolivia, Brazil, Paraguay]

49. Decimiana rehni (Chopard, 1913) [Argentina, Bolivia, Brazil, Paraguay]

50. Decimiana tessellata (Charpentier, 1841) [Brazil, Argentina, Paraguay, Uruguay]

51. Decimiana elliptica Menezes and Bravo, 2012 [Brazil]

\section{Genus: Lagrecacanthops Roy, 2004}

52. Lagrecacanthops brasiliensis Roy, 2004 [Brazil]

53. Lagrecacanthops guyanensis Roy, 2004 [French Guiana]

\section{Genus: Metilia Stal, 1877}

54. Metilia boliviana (Werner, 1927) [Bolivia]

55. Metilia brunnerii (Saussure, 1871) [Bolivia, Brazil, Colombia, Costa Rica, Ecuador, French Guiana, Nicaragua, Peru, Surinam, Venezuela]

56. Metilia adusta (Gerstaecker, 1889) [Costa Rica, Peru]

57. Metilia integra Stål, 1877 [Brazil]

58. Metilia coloradensis (Salazar, González \& Miller, 2012) [Brazil, Colombia, French Guiana]

59. Metilia septemspinosa (Ippolito, 2007) [Brazil, Ecuador]

60. Metilia caina Rafael, 2014 [Brazil, Colombia, French Guiana]

61. Metilia glabripenis Rafael, 2014 [Brazil, French Guiana]

62. Metilia guttata Rafael, 2014 [Brazil]

63. Metilia pinima Rafael, 2014 [Peru, Venezuela]

64. Metilia vulgaris Rafael, 2014 [Brazil, Peru]

65. Metilia yutoensis Rafael, 2014 [Colombia]

\section{Genus: Miracanthops Roy, 2004}

66. Miracanthops eseejja Rivera, 2005 [Peru]

67. Miracanthops lombardoi Roy, 2004 [Ecuador, Peru]

68. Miracanthops occidentalis (Lombardo \& Ippolito, 2004) [Ecuador, Peru]

69. Miracanthops poulaini Roy, 2004 [Peru, Ecuador]

\section{Genus: Pseudacanthops Saussure, 1870}

70. Pseudacanthops angulata (Lichtenstein, 1802) [Surinam]

71. Pseudacanthops caelebs (Saussure, 1869) [Bolivia, Belize, Brazil, Guatemala, Honduras, Mexico, Nicaragua, Venezuela]

72. Pseudacanthops lobipes La Greca \& Lombardo, 1997 [Bolivia]

73. Pseudacanthops spinulosa Saussure, 1870 [Bolivia, Brazil, Colombia, Ecuador, French Guiana, English Guiana, Venezuela]

74. Pseudacanthops centralis Lombardo, Ippolito \& Rivera, 2013 [Nicaragua, Panama]

75. Pseudacanthops clorindae Lombardo, Ippolito \& Rivera, 2013 [Brazil, Peru]

76. Pseudacanthops huaoranianus Lombardo, Ippolito \& Rivera, 2013 [Equador]

\section{Subfamily: Acontistinae \\ 1. Tribe: Acontistini \\ 13. Genus: Acontista Saussure, 1842}

77. Acontista amazonica Beier, 1929 [Brazil]

78. Acontista amoenula Gerstaecker, 1889 [Brazil, Peru]

79. Acontista aurantiaca (Burmeister, 1838) [Paraguay] 
Updated Checklist and Global Diversity of Chaeteessidae, Mantoididae, Metallyticidae, Acanthopidae, Amorphoscelididae and Sibyllidae (Mantodea: Insecta)

80. Acontista bolivari Giglio-Tos, 1915 [Bolivia, Brazil]

81. Acontista brevipennis Saussure, 1872 [Argentina, Bolivia, Brazil, Paraguay]

82. Acontista cayennensis Saussure \& Zehntner, 1894 [Brazil, French Guiana]

83. Acontista championi Kirby, 1904 [Brazil, Guatemala]

84. Acontista chopardi Giglio-Tos, 1927 [French Guiana]

85. Acontista concinna (Perty, 1833) [Argentina, Bolivia, Brazil, Colombia, Ecuador, Peru, Paraguay, French Guiana]

86. Acontista cordillerae (Saussure, 1869) [Brazil, Colombia, Costa Rica, French Guiana, Mexico, Panama, Surinam]

87. Acontista cubana De Zayas, 1974 [Cuba]

88. Acontista ecuadorica Hebard, 1924 [Ecuador]

89. Acontista eximia Pascoe, 1882 [Brazil, Panama]

90. Acontista festae Giglio-Tos, 1915 [Ecuador]

91. Acontista fraterna Saussure \& Zehntner, 1894 [Costa Rica, Mexico]

92. Acontista gracilis Chopard, 1911 [French Guiana]

93. Acontista inquinata Saussure \& Zehntner, 1894 [Mexico]

94. Acontista iriodes Hebard, 1919 [Colombia]

95. Acontista maroniensis Chopard, 1911 [Brazil, French Guiana]

96. Acontista mexicana (Saussure \& Zehntner, 1871) [Brazil, Costa Rica, Guatemala, Mexico, Netherlands Antilles, Nicaragua, Panama]

97. Acontista minima Giglio-Tos, 1915 [Colombia, Trinidad]

98. Acontista multicolor (Saussure, 1870) [Argentina, Antilles, Colombia, Nicaragua, Trinidad \& Tobago, Uruguay, Venezuela]

99. Acontista parva Beier, 1942 [Brazil]

100. Acontista piracicabensis Toledo Piza, 1967 [Brazil]

101. Acontista rehni Giglio-Tos, 1927 [Brazil, Costa Rica, Puerto Rica, Paraguay]

102. Acontista semirufa Westwood, 1889 [Brazil]

103. Acontista vitrea Saussure \& Zehntner, 1894 [Brazil, Colombia, Costa Rica, Ecuador, Mexico, Panama]

\section{Genus: Callibia Stal, 1877}

104. Callibia diana (Stoll, 1813) [Bolivia, Brazil, Colombia, Ecuador, French Guiana, Peru, Venzuela]

\section{Genus: Paratithrone Lombardo, 1996}

105. Paratithrone royi Lombardo, 1995 [Brazil, Colombia, Equador, French Guiana]

\section{Genus: Raptrix Terra, 1995}

106. Raptrix intermedia Lombardo \& Marletta, 2004 [Brazil, Ecuador, French Guiana]

107. Raptrix occidentalis Lombardo \& Marletta, 2004 [Bolivia, Brazil, Ecuador, French Guiana, Peru]

108. Raptrix perspicua (Fabricius, 1787) [Brazil, Colombia, Ecuador, French Guiana, Surinam, Venezuela]

109. Raptrix westwoodi (Saussure \& Zehntner, 1894) [Brazil, Colombia, Costa Rica]

\section{Genus: Tithrone Stal, 1877}

110. Tithrone catharinensis Toledo Piza, 1961 [Brazil]

111. Tithrone clauseni Jantsch, 1995 [Arizona-USA]

112. Tithrone corseuili Jantsch, 1986 [California-USA]

113. Tithrone laeta Lombardo, 1996 [Venezuela]

114. Tithrone latipennis Lombardo, 1996 [Colombia, Ecuador]

115. Tithrone major Toledo Piza, 1961 [Brazil]

116. Tithrone roseipennis (Saussure, 1870) [Brazil, Colombia, Costa Rica, French Guiana, Peru, Trinidad \& Tobago, Venezuela]

\section{Subfamily: Stenophyllinae \\ 1. Tribe: Stenophyllini}

18. Genus: Stenophylla Westwood, 1845

117. Stenophylla cornigera Westwood, 1843 [Brazil, Venezuela]

118. Stenophylla gallardi Roy, 2005 [French Guiana]

119. Stenophylla lobivertex Lombardo, 2000 [Amazonia, Colombia, Ecuador, Peru] 


\section{Family: Amorphoscelididae \\ 1. Subfamily: Amorphoscelinae \\ 1. Tribe: Amorphoscelini \\ 19. Genus: Amorphoscelis Stal, 1871}

120. Amorphoscelis abyssinica Giglio-Tos, 1913 [Ethiopia, Somalia, Tanzania]

121. Amorphoscelis angolica Beier, 1969 [Angola]

122. Amorphoscelis annulicornis Stal, 1871[India-NE, Malayasia, Melaka, Nepal, Sri Lanka]

123. Amorphoscelis asymmetrica Ingrisch, 1999 [Yemen]

124. Amorphoscelis austrogermanica Werner, 1923 [Namibia, Natal, Transvaal, Tanzania]

125. Amorphoscelis bimaculata Roy, 2010 [Sabah]

126. Amorphoscelis borneana Giglio-Tos, 1914 [Malaysia, Borneo]

127. Amorphoscelis brunneipennis Beier, 1956 [India, Sri Lanka]

128. Amorphoscelis chinensis Tinkham, 1937 [China]

129. Amorphoscelis chopardi Roy, 1962 [Ivory Coast, Ghana]

130. Amorphoscelis elegans Giglio-Tos, 1914 [Ghana, Guinea]

131. Amorphoscelis griffinii Giglio-Tos, 1913 [Ivory Coast, Cameroon, Gabon]

132. Amorphoscelis grisea Bolivar, 1908 [Ivory Coast, Cameroon, Guinea, Congo, Gabon,

Uganda]

133. Amorphoscelis hainana Yang, 2002 [China]

134. Amorphoscelis hamata Roy, 2009 [Kenya]

135. Amorphoscelis huismani Roy, 2010 [Sabah]

136. Amorphoscelis javana Roy, 1966 [Java]

137. Amorphoscelis kenyensis Stiewe, 2009 [Kenya, Somalia]

138. Amorphoscelis lamottei Roy, 1963 [Congo, Gabon, Ghana, Guinea, Ivory Coast, Tanzania, Uganda]

139. Amorphoscelis laxeretis Karsch, 1894 [Congo, Gabon, Ghana, Ivory Coast, Sierra Leone]

140. Amorphoscelis machadoi Beier, 1969 [Angola]

141. Amorphoscelis morini Roy, 2013 [Congo]

142. Amorphoscelis naumanni Kaltenbach, 1983 [Afganistan]

143. Amorphoscelis nigriventer Beier, 1930 [Ivory Coast, Guinea, Ghana]

144. Amorphoscelis nubeculosa Werner, 1908 [Cameroon]

145. Amorphoscelis opaca Bolivar, 1908 [Cameroon]

146. Amorphoscelis orientalis Giglio-Tos, 1914 [Kenya, Somalia, Tanzania]

147. Amorphoscelis pallida Giglio-Tos, 1914 [Cameroon, Kenya, Nigeria]

148. Amorphoscelis pantherina Roy, 1966 [Iraq, Turkey]

149. Amorphoscelis papua Werner, 1923 [Indonesia, New Guinea]

150. Amorphoscelis parva Beier, 1952 [Sumba]

151. Amorphoscelis pellucida Westwood, 1889 [Australia, Java, Singapur, Sri Lanka]

152. Amorphoscelis phaesoma Yang, 1999 [China]

153. Amorphoscelis philippina Werner, 1926 [Philippines]

154. Amorphoscelis pinheyi Roy, 2007 [Mozambique]

155. Amorphoscelis pulchella Giglio-Tos, 1914 [Angola, Congo, Kenya, Tanzania, Uganda, Zimbabwe]

156. Amorphoscelis pulchra Bolivar, 1908 [Congo, Ivory Coast, Gabon, Ghana, Cameroon, Sierra Leone, Uganda]

157. Amorphoscelis punctata Roy, 1962 [Ethiopia, Eritrea]

158. Amorphoscelis reticulata Werner, 1933 [ Sarawak-Borneo]

159. Amorphoscelis rufula Roy, 1966 [Borneo, Malaysia]

160. Amorphoscelis siebersi Werner, 1933 [Borneo]

161. Amorphoscelis singaporana Giglio-Tos, 1915 [India, Borneo, Cambodia, Java, Singapore, Sumatra, Thailand, Vietnam]

162. Amorphoscelis spinosa Beier, 1942 [Sri Lanka]

163. Amorphoscelis stellulatha Yang, 1999 [China]

164. Amorphoscelis subnigra Werner, 1933 [Borneo]

165. Amorphoscelis sulawesiana Roy, 2010 [Sulawesi Tengah]

166. Amorphoscelis sumatrana Roy, 2010 [Malayasia, Sumatra] 
Updated Checklist and Global Diversity of Chaeteessidae, Mantoididae, Metallyticidae, Acanthopidae, Amorphoscelididae and Sibyllidae (Mantodea: Insecta)

167. Amorphoscelis tigrina Giglio-Tos, 1914 [Benin, Guinea, Cameroon, Nigeria, Senegal, Ivory Coast, Burkina Faso, Sudan]

168. Amorphoscelis tuberculata Roy, 1963 [Malawi, Mozambique, Namibia, Tanzania, Tramsvaal, Zimbabwe]

169. Amorphoscelis villiersi Roy, 1984 Congo]

20. Genus: Bolivaroscelis Roy, 1973

170. Bolivaroscelis bolivarii (Giglio-Tos, 1913) [Cameroon, Congo, Gabon]

171. Bolivaroscelis carinata (Bolivar, 1908) [Cameroon, Gabon]

172. Bolivaroscelis werneri (Roy, 1962) [Cameroon, Ghana]

\section{Genus: Caudatoscelis Roy, 1973}

173. Caudatoscelis annulipes (Karsch, 1892) [Bioko, Congo, Ghana]

174. Caudatoscelis caudata (Giglio-Tos, 1914) [Congo, Gabon]

175. Caudatoscelis collarti (Roy, 1964) [Congo, Ghana, Uganda]

176. Caudatoscelis lagrecai (Roy, 1964) [Ghana, Nigeria]

177. Caudatoscelis marmorata (Roy, 1965) [Ghana, Ivory Coast, Nigeria]

\section{Genus: Gigliotoscelis Roy, 1973}

178. Gigliotoscelis simulans (Giglio-Tos, 1913) [Ghana, Guinea, Congo, Cameroon, Ivory Coast, Gabon, Togo]

\section{Genus: Maculatoscelis Roy, 1973}

179. Maculatoscelis ascalaphoides (Bolivar, 1908) [Angola, Ghana, Guinea, Cameroon, Congo, Tanzania]

180. Maculatoscelis gilloni (Roy, 1964) [Ivory Coast]

181. Maculatoscelis maculata (Roy, 1965) [Ivory Coast, Ghana]

\section{Subfamily: Paraoxypilinae \\ 1. Tribe: Paraoxypilini \\ 24. Genus: Cliomantis Giglio-Tos, 1913}

182. Cliomantis cornuta Giglio-Tos, 1913 [Australia]

183. Cliomantis dispar Tindale, 1923 [Australia]

184. Cliomantis lateralis Hinton, 1939 [Queensland]

185. Cliomantis obscura Hinton, 1939 [Queensland]

25. Genus: Exparoxypilus Beier, 1929

186. Exparoxypilus africanus Beier, 1929 [Zanzibar]

\section{Genus: Gyromantis Giglio-Tos, 1913}

187. Gyromantis kraussii (Saussure, 1872) [Australia, Neuholland]

188. Gyromantis occidentalis Sjostedt, 1918 [Northwest Australia]

\section{Genus: Metoxypilus Giglio-Tos, 1913}

189. Metoxypilus costalis (Westwood, 1889) [New Guinea]

190. Metoxypilus lobifrons (Stal, 1877) [Queensland]

191. Metoxypilus werneri Beier, 1929 [Kai Islands]

28. Genus: Myrmecomantis Giglio-Tos, 1913

192. Myrmecomantis atra Giglio-Tos, 1913 [Queensland]

\section{Genus: Nesoxypilus Beier, 1965}

193. Nesoxypilus albomaculatus (Werner, 1933) [Australia] 194. Nesoxypilus pseudomyrmex Milledge, 1990 [Australia]

\section{Genus: Paraoxypilus Saussure, 1870}

195. Paraoxypilus armatus Giglio-Tos, 1913 [Thursday Island-Queensland]

196. Paraoxypilus distinctus Beier, 1929 [Australia] 
197. Paraoxypilus flavifemur Sjostedt, 1918 [Queensland] 198. Paraoxypilus insularis Tindale, 1923 [Australia] 199. Paraoxypilus kimberleyensis Sjostedt, 1918 [Australia] 200. Paraoxypilus laticollis Tindale, 1923 [Australia] 201. Paraoxypilus tasmaniensis Saussure, 1870 [Tasmania] 202. Paraoxypilus verreauxii Saussure, 1870 [Tasmania]

\title{
31. Genus: Phthersigena Stal, 1871
}

203. Phthersigena centralis Giglio-Tos, 1915 [Australia] 204. Phthersigena conspersa Stal, 1871 [Australia, New Guinea] 205. Phthersigena insularis Beier, 1965 [Australia] 206. Phthersigena melania (Tindale, 1923) [Australia] 207. Phthersigena minor Sjostedt, 1918 [Australia] 208. Phthersigena nebulosa (Sjostedt, 1918) [Australia] 209. Phthersigena pallidifemur Tindale, 1923 [Australia] 210. Phthersigena timorensis Beier, 1952 [Timor] 211. Phthersigena unicornis (Tindale, 1923) [Australia]

\section{Subfamily: Perlamantinae \\ 1. Tribe: Perlamantini \\ 32. Genus: Paramorphoscelis Werner, 1907}

212. Paramorphoscelis gondokorensis Werner, 1907 [Angola, Burkina Faso, Ghana, Guinea, Nigeria, Senegal, Sudan, Uganda]

\section{Genus: Perlamantis Guerin-Meneville, 1843}

213. Perlamantis algerica Giglio-Tos, 1914 [Algeria]

214. Perlamantis allibertii Guerin-Meneville, 1843 [France, Spain, Algeria, Morocco, Libea, Tunesia]

\author{
6. Family: Sibyllidae \\ 1. Subfamily: Sibyllinae \\ 1. Tribe: Sibyllini \\ 34. Genus: Leptosibylla Roy, 1996
}

215. Leptosibylla gracilis Roy, 1996 [Cameroon, Central Africa Republic]

35. Genus: Presibylla Bolivar, 1908

216. Presibylla elegans (Bolivar, 1908) [Cameroon, Congo, Gabon]

217. Presibylla speciosa Roy, 1996 [Cameroon, Nigeria]

\section{Genus: Sibylla Stål, 1856}

218. Sibylla (Sibylla) dives Giglio-Tos, 1915 [Angola, Congo, Tanzania, Uganda, Malawi, Zambia, Zimbabwe]

219. Sibylla (Sibylla) dolosa Roy, 1975 [Ghana]

220. Sibylla (Sibylla) gratiosa Rehn, 1912 [Congo, Ivory Coast, Guinea, Gabon, Ghana]

221. Sibylla (Sibylla) limbata Giglio-Tos, 1915 [Cameroon, Congo, Gabon, Ivory Coast, Ghana]

222. Sibylla (Sibylla) maculosa Roy, 1996 [Cameroon, Congo, Gabon]

223. Sibylla (Sibylla) marmorata Roy, 1996 [Cameroon, Republic of Central Africa]

224. Sibylla (Sibylla) polyacantha Gerstaecker, 1889 [Congo]

225. Sibylla (Sibylla) pretiosa Stal, 1856 [Abyssinia, Ethiopia, Kenya, Tanzania, Uganda,

Malawi, Somalia, Zambia, Namibia, Republic South Africa, Swaziland, Cameroon, Congo, Zimbabwe]

226. Sibylla (Sibyllopsis) griffinii griffinii Giglio-Tos, 1915 [Benin, Cameroon, Ivory Coast, Gabon, Ghana, Guinea, Nigeria, Tongo]

227. Sibylla (Sibyllopsis) griffinii guineensis Roy, 1965 [Guinea, Ivory Coast, Liberia, Syria] 228. Sibylla (Sibyllopsis) operosa Roy, 1996 [Syria, Liberia, Guinea, Ghana, Nigeria, Ivory Coast]

229. Sibylla (Sibyllopsis) pannulata Karsch, 1894 [Cameroon, Congo, Gabon, Guinea, Nigeria, Republic Central Africa, Zaire]

230. Sibylla (Sibyllopsis) punctata Roy, 1996 [Cameroon, Republic Central Africa, Zaire]

231. Sibylla (Sibyllopsis) vanderplaetseni Roy, 1963 [Congo, Gabon, Ghana, Cameroon, Guinea, Ivory Coast, Republic of Central Africa] 


\section{CONCluSiON}

The distribution pattern of six families of Mantodea: Chaeteessidae, Mantoididae, Metallyticidae, Acanthopidae, Amorphoscelididae and Sibyllidae demonstrated that most of the species belongs to Neotropical ecozone of the world, i.e. countries of Central and South America; and Africa and SouthEast Asia. Out of 231 valid species of these families, only 5 belong to India while 67 belong to Brazil.

\section{REFERENCES}

[1] Otte, D., Spearman, L. and Stiewe, M.B.D., Mantodea Species File Online. Version 5.0/5.0. [retrieved on 15.10.2016] . http://Mantodea.SpeciesFile.org (2016).

[2] Prete, F.R. and Mahaffey, R.J., Appetitive responses to computer-generated visual stimuli by the praying mantis Sphodromantis lineola (Burr.). Visual Neurosc. 10(4), 669-679 (1993).

[3] Prete, F.R. and Wolfe, M.M., Religious supplicant, seductive cannibal, or reflex machine? In search of the praying mantis. J. Hist. Biol. 25, 91-136 (1992).

[4] Sampaio, M.V., Bueno, V.H.P., Silveira, L.C.P. and Auad, A.M., Biological control of insect pests in the Tropics. Encyclopedia of Life Support Systems (EOLSS). Oxford: EOLSS Publishers (2008).

[5] Wang, T., Synopsis on the classification of Mantodea from China. Shanghai Scientific and Technological, Literature Publishing House, Shanghai, pp. 176 (1993).

[6] Kaltenbach, A.P., Unterlagen für eine Monographie der Mantodea des südlichen Afrika: 1. Artenbestand, geographische Verbreitung und Ausbreitungsgrenzen (Insecta: Mantodea). Ann. Naturhist. Mus. Wien 98 B, 193-346 (1996).

[7] Klass, K.-D. and Ehrmann, R.,13. Ordnung Mantodea, Fangschrecken, Gottesanbeterinnen. In: Dathe, H. H. (ed.): Lehrbuch der Speziellen Zoologie, Band I: Wir-bellose Tier, 5. Teil: Insecta. Heidelberg, Berlin (Spektrum Akademischer Verlag), pp. 182-197 (2003).

[8] Rivera, J., A historical review of praying mantid taxonomy and systematics in the Neotropical Region: State of knowledge and recent advances (Insecta: Mantodea). Zootaxa 2638, 44-64 (2010).

[9] Schwarz, C.J. and Konopik, O., An annotated checklist of the praying mantises (Mantodea) of Borneo, including the results of the 2008 scientific expedition to Lanjak Entimau Wildlife Sanctuary, Sarawak. Zootaxa 3797, 130-168 (2014).

[10] Shcherbako, E.O. and Savitsky, V.Yu., New data on the fauna, taxonomy and ecology of praying mantises (Dictyoptera, Mantodea) from Russia. Ent. Rev. 95(2), 181-199 (2015).

[11] Svenson, G.J. and Whiting, M.F., Phylogeny of Mantodea based on molecular data: evolution of a charismatic predator. Syst. Ent. 29, 359-370 (2004).

[12] Wieland, F., Ph. D. thesis, University of Göttingen, Germany, pp. 306+Appendix pp. 29 (2010).

[13] Wieland, F., The phylogenetic system of Mantodea (Insecta: Dictyoptera). Species, Phylogeny and Evol. 3(1), 3-222 (2013).

[14] Legendre, F., Nel, A., Svenson, G.J., Robillard, T., Pellens, R. and Grandcolas, F., Phylogeny of Dictyoptera: dating the origin of cockroaches, praying mantises and termites with molecular data and controlled fossil evidence. PLoS ONE, 10, e0130127. DOI: 10.1371/journal.pone.0130127 (2015).

[15] Rivera, J. and Svenson, G.J., The Neotropical 'polymorphic earless praying mantises' - Part I: molecular phylogeny and revised higher-level systematics (Insecta: Mantodea, Acanthopoidea). Syst. Ent. 41, 607-649. DOI: 10.1111/syen.12178 (2016).

[16] Serville, J.G.A., Revue méthodique des Insectes de l'ordre des Orthoptères. Ann. Sci. Nat. 22, 28-65 (1831).

[17] Burmeister, H.C., Handbuch der Entomologie. Fangschrecken, Mantodea. Handbuch der Entomologie, Vol. 2, v-viii. Theodor Christian Friedrich Enslin, Berlin, (1838).

[18] De Haan, W., Bijdragen tot de Kennis der Orthoptera Zoologie. Verhandel. Natuurl. Gesch. Overzee. Bezittingen 2, 45-248 (1842).

[19] de Saussure, H., Essai d'un système des Mantides. Mitt. Schweiz. Ent. Gesell. 3, 49-73 (1869).

[20] de Saussure, H., Additions au système des Mantides. Mitt. Schweiz. Ent. Gesell. 3, 221-244 (1870). 
[21] Stål, C., Recherches sur le système des Mantides. Bihang till Kongliga Svenska VetenskapsAkademiens Handlingar 1, 1-26 (1873).

[22] Stål, C., Systema mantodeorum. Essai d'une systematization nouvelle des Mantodées. Bihang till Kongliga Svenska Vetenskaps-Akademiens Handlingar 4, 1-91 (1877).

[23] Kirby, W.F., A Synonymic Catalogue of Orthoptera (Forficulidae, Hemimeridae, Blattidae, Mantidae and Phasmidae). British Mus., Nat. Hist., London 1, 1-10 (1904).

[24] Giglio-Tos, E., Saggio di una nuova classificazione dei Mantidi. Bull. Soc. Ent. Ital. 49, 50-87 (1919).

[25] Giglio-Tos, E., Orthoptera Mantidae. Das Tierreich 50. Berlin and Leipzig: Walter de Gruyter and Co., pp. 707 (1927).

[26] Handlirsch, A., Mantodea. Handbuch der Entomologie. Geschichte, Literatur, Technik, Paläontol., hylogen. Syst. 3(4-8), 493-502 (1925).

[27] Chopard, L., Ordre des Dictyoptères. Sous-Ordre des Mantodea Burmeister, 1838. Traité Zool. Anat. System. Biol. 9, 386-407 (1949).

[28] Beier, M., Blattopteroidea-mantodea. Bronn's Klassen und Ordnungen des Tierreichs, Vol. 5 Insecta-Arthropoda, Part III, Book 5, No. 5 (ed. by H.G. Bronn), Akademische Verlagsgesellschaft Geest and Portig, Leipzig, pp. 850-970 (1964).

[29] Beier, M., Mantodea (Fangheuschrecken). Handbuch der Zoologie (ed. by J. G. Helmcke, D. Starck and H.Wermuth), Vol. 4(2) 2/12, pp. 1-47. Walter de Gruyter and Co, Berlin. (1968).

[30] Roy, R., General observations on the systematics of Mantodea. Evolutionary Biology of Orthopteroid Insects (ed. by B. Baccetti), Halsted Press, John Wiley and Sons, New York, New York, pp. 489-495 (1987).

[31] Roy, R., Morphology and taxonomy. The Praying Mantids (ed. by F.R. Prete, H. Wells, P.H. Wells and L.E. Hurd). The Johns Hopkins University Press, Baltimore, Maryland and London, pp. 19-40 (1999).

[32] Ehrmann, R., Systematik der Ordnung Mantoptera (Mantodea) (Insecta: Dictyoptera). Arthropoda 5, 6-12 (1997).

[33] Ehrmann, R., Mantodea Gottesanbeterinnen der Welt. Münster: Natur und Tier-Verlag, pp. 519 (2002).

[34] Otte D. and Spearman L., Mantid Species File. Catalog of the Mantids of the World. Insect Diversity Association, Publication No. 1, pp. 489 (2005).

[35] Svenson, G.J., Hardy, N.B., Cahill Wightman, H.M. and Wieland, F., On flowers and twigs: phylogenetic revision of the plant-mimicking praying mantises (Mantodea: Empusidae and Hymenopodidae) with a new generic classification. Syst. Ent. 40, 789-834 (2015).

[36] Svenson, G.J., Medellín, C. and Sarmiento, C., Re-evolution of a morphological precursor of crypsis investment in the newly revised horned praying mantises (Insecta, Mantodea, Vatinae). Syst. Ent. 41, 229-255 (2016).

[37] Patel, S. and Singh, R., Updated Checklist and Distribution of Mantidae (Mantodea : Insecta) of the World. Internat. J. Res. St. Zool. (IJRSZ) 2(4), 1-46 (2016).

[38] Salazar, J.A., Notas sobre Metallyticus Westwood, 1837; Chaeteessa Burmeister, 1838 у Mantoida Newman, 1838. Tres géneros primitivos de mantidos tropicales (Dictyoptera: antodea). Lambillionea 2, 265-276 (2005).

[39] Grimaldi, D.A., A revision of Cretaceous mantises and their relationships, including new taxa (Insecta: Dictyoptera: Mantodea). Amer. Mus. Novit. 3412, 1-47 (2003).

[40] Zherikhin V.V., Order Mantida Latreille, 1802. The mantises (=Mantodea Burmeister, 1838). In: Rasnitsyn A.P. and Quicke D.L.J. (Eds.): History of Insects. Kluwer, Dodrecht, 273-276 (2002).

[41] Svenson, G.J. and Whiting, M.F., Reconstructing the origins of praying mantises (Dictyoptera, Mantodea): The roles of Gondwanan vicariance and morphological convergence. Cladistics 25(5), 468-514 (2009).

[42] Grimaldi, D.A. and Engel, M.S., Evolution of the Insects. Cambridge University Press, New York, 784 pp. (2005). 
Updated Checklist and Global Diversity of Chaeteessidae, Mantoididae, Metallyticidae, Acanthopidae, Amorphoscelididae and Sibyllidae (Mantodea: Insecta)

[43] La Greca, M. and Lombardo, F., Mantodei Neotropicali. I. Il genere Mantoida descrizione di due nuove specie. Animalia 16, 55-67 (1989).

[44] Roy, R., Contribution à la connaissance du genre néotropical Mantoida Newman, 1838 (Dict., Mantoididae). Bull. Soc. Ent. Fr. 115(1), 22 (2010).

[45] Wieland, F. and Schütte, K., Unrecognized museum specimen expands distribution of Mantoida (Insecta: Mantodea) into the central Caribbean. Ent. Itteil. Zool. Mus. Hamburg 15(186), 305314 (2011).

[46] Agudelo, R.A.A., A new genus and species of Mantoididae (Mantodea) from the Brazilian and Venezuelan Amazon, with remarks on Mantoida Newman, 1838. Zootaxa (3797),194-206. doi: 10.11646/zootaxa.3797.1.14. (2014)

[47] Wieland, F., The genus Metallyticus reviewed (Insecta: Mantodea). Species, Phylogeny and Evolution 1(3), 147-170 (2008).

[48] Robinson, M.H., Defenses against visually hunting predators. Evolutionary Biology, Vol. III (ed. by T. Dobzhansky,M.K. Hecht and W.C. Steere), Meredith Corp, New York, NewYork, pp. 225-259 (1969).

[49] Robinson, M.H. and Robinson, B., By dawn's early light: matutinal mating and sex attractants in a Neotropical mantid. Science 205, 825-827 (1979).

[50] Edmunds, M. and Brunner, D., Ethology of defenses against predators. The Praying Mantids (ed. by F.R. Prete, H. Wells, P.H. Wells and L.E. Hurd), The Johns Hopkins University Press, Baltimore, Maryland and London, pp. 276-299 (1999).

[51] Agudelo, R.A.A., Lombardo, F. and Jantsch, L.J., Checklist of the Neotropical mantids (Insecta, Dictyoptera, Mantodea), Biota Colombiana 8 (2), 105-158 (2007).

[52] Lombardo, F., A review of the genus Decimiana Uvarov, 1940 (Insecta: Mantodea), with description of a new species. Proc. Acad. Nat. Sci. Philad. 150, 159-171 (2000).

[53] Agudelo, R.A.A., Lombardo, F. and Jantsch, L.J., Checklist of the neotropical mantids (Insecta, Dictyoptera, Mantodea). Biota Colombiana 8, 105-158 (2007).

[54] Lombardo, F. and Ippolito, S., Revision of the species of Acanthops Serville, 1831 (Mantodea, Mantidae, Acanthopinae) with comments on their Phylogeny. Ann. Ent. Soc. Amer. 97, 10761102 (2004).

[55] Roy, R, Lagrecacanthops et Miracanthops, deux nouveaux genres d'Acanthopinae (Dictyoptera, Mantodea, Acanthopidae). Bull. Soc. Ent. France, 109, 491-498 (2004).

[56] Menezes, E.d.C. and Bravo, F., A new species of Margaromantis Piza, 1982 (Insecta: Mantodea) from Brazil. Biodiver. Data J. 3, 1-9 (2015).

[57] Lombardo, F., Ippolito, S. and Rivera, J., Synopsis of the Neotropical mantid genus Pseudacanthops Saussure, 1870, with the description of three new species (Mantodea: Acanthopidae). Rev. Suis. Zool. 120, 373-403 (2013).

[58] Arteaga, B.L.A., Parra, G.A.C., Medellín, R.M.C., Martínez, H.N.J., Mantidofauna (Insecta : Mantodea ) en fragmentos de Bosque seco Tropical (BS-T) en el Departamento del Atlantico (Colombia). Bol. Cient. Mus. Hist. Nat. 18 (2), 243-262 (2014).

[59] Esteves, L. and Mendes, L.F., Mantodea do Centro de Zoologia I.- Amorphoscelidae. Garcia de Orta, Sér. Zool. 23 (1), 93-97, (1999)

[60] Roy, R., Une nouvelle espèce africaine d’Amorphoscelis Stål, 1871 (Dictyoptera Mantodea). Bull. Soc. Ent. France 112 (3), 387-388 (2007).

[61] Roy, R. and Stiewe, M.B.D. (2009). Contribution to the knowledge of Eastern African Amorphoscelis Stål, 1871, (Dictyoptera, Mantodea, Amorphoscelidae) with description of two new species. Bulletin de la Société entomologique de France, 114 (2), 2009 : 195-209.

[62] Roy, R., Révision des Sibyllinae (Mantodea). Bull. Mus. Nat. Hist. Nat., Paris 4e ser. 18, Section A, nos 1-2, 69-138 (1996).

[63] Mukherjee, T.K., Ehrmann, R. and Chatterjee, P., Checklist of Mantodea (Insecta) from India. Priamus (Serial Publication of the Centre for Entomological Studies Ankara), (Suppl.) 30, 1-61 (2014). 
[64] Ehrmann, R. \& Borer, M. (2015). Mantodea (Insecta) of Nepal: an annotated checklist. In Hartmann, M. \& J. Weipert: Biodiversität \& Naturausstattung im Himalaya, Bd. Vietnam, pp. 227-274.

[65] Agudelo, R.A.A., Mántidos de Colombia (Dictyoptera: Mantodea). In: Fernández C, Gonzalo Andrade-C FM, Amat GGD (Eds) Insectos de Colombia. Universidad Nacional, Facultad de Ciencias, Bogota, pp. 43-60 (2004).

[66] Ehrmann, R., Mantodea from Turkey and Cyprus (Dictyoptera: Mantodea), Articulata 26(1), 142 (2011).

[67] Paulian, R., Faune de Madagascar. V. Insectes Mantodea. Publications de l'Institut de Recherche Scientifique Tananarive - Tsimbazaza 5, 1-102 (1957).

[68] Ragge, D.R. and Roy, R., A review of praying mantises of Ghana (Dictyoptera, Mantodea). Bull. l'Inst. Fond. d'Afriq. Noire (IFAN) Série A 29(2), 586-644 (1967).

[69] Beier, M., Mantodea. Subfamilie: Hymenopodidae. Genera Insectorum, Fasc. 196 (ed. by P. Wytsman), pp. 1-37 (1934).

[70] Beier, M., Mantodea. Subfamilie: Thespidae. Genera Insectorum, Fasc. 200 (ed. by P. Wytsman), pp. 1-32 (1935).

[71] Beier, M. Mantodea. Subfamilie: Mantinae. Genera Insectorum, Fasc. 203 (ed. by P. Wytsman), pp. 1-146 (1935).

[72] Beier, M., Blattopteroidea-mantodea. Bronn's Klassen und Ordnungen des Tierreichs, Vol. 5 Insecta-Arthropoda, Part III, Book 5, No. 5 (ed. by H.G. Bronn), Akademische Verlagsgesellschaft Geest and Portig, Leipzig, pp. 850-970 (1964).

[73] Beier, M., Insects of Micronesia, Mantodea. Insects Micronesia 5(2), 173-175 (1972).

[74] Roy, R. and Svenson, G., Revision of the genus Ceratomantis Wood-Mason, 1876 (Dictyoptera: Mantidae). Bull. Soc. Ent. Fr. 112(4), 433-444 (2007).

[75] Lombardo, F. and Agabiti, B., The mantids of Ecuador, with some biogeographic considerations. J. Orthoptera Res. 10, 89-104 (2001).

[76] Salazar, J.A., Liste de los Mantodea conocidos para Colombia (Insecta). Biota Colomb. 3(1), 119-130 (2002).

[77] Rivera, J., Apuntes sobre algunas especies de Photininae de Perú, incluyendo la descripción de una nueva especie de Orthoderella Giglio-Tos, 1897 (Dictyoptera: Mantodea: Mantidae). Rev. Peru. Ent. 43, 7-12 (2003).

[78] Roy, R., A historical review of nomenclature and high-level classification of praying mantises (Mantodea), including a provisional checklist of the names associated to suprageneric ranks. Zootaxa 3797, 9-28 (2014).

[79] Agudelo, A.A. and Rivera, J., Some taxonomic and nomenclatural changes in American Mantodea (Insecta, Dictyoptera), Part I. Zootaxa 3936 (3), 335-356 (2015).

[80] Maldaner, C., Revisão taxonômica de Metilia Stål, 1877 (Mantodea: Acanthopidae) e descrição de um gênero novo. Dissertação (Mestrado), INPA, Manaus, pp. 115 (2014). 
Updated Checklist and Global Diversity of Chaeteessidae, Mantoididae, Metallyticidae, Acanthopidae, Amorphoscelididae and Sibyllidae (Mantodea: Insecta)

\section{AUTHORS' BIOGRAPHY}

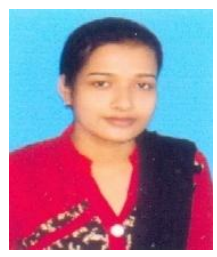

Miss Shveta Patel, is a Ph. D. scholar working on biosystematic studies of mantids in northeastern Uttar Pradesh. She has presented papers in several national and international seminars and symposia and published 4 research papers.

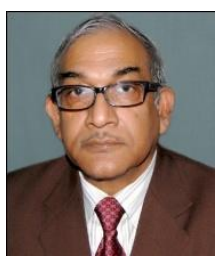

Dr. Rajendra Singh, is Professor \& Head of the Department of Zoology, Former Head of Department of Biotechnology, D.D.U. Gorakhpur University, Gorakhpur and has 38 years of experience in the fields of bioecology and biosystematics of aphids, their parasitoids and predators, spiders, and soil arthropods and published about 200 research articles of international repute, authored 6 text/reference books, supervised one D.Sc. and $26 \mathrm{Ph}$. D. students, and completed 12 research projects. He is fellow/life member of several scientific organizations/societies, Chief Editor of Journal of Aphidology and is on the editorial/reviewer panel of several International Journals. Prof. Singh also served in the university as Pro-Vice Chancellor. 